\title{
Modeling of Piezoelectric Energy Harvesting from Freely Oscillating Cylinders in Water Flow
}

\author{
Min Zhang, ${ }^{1,2}$ YingZheng Liu, ${ }^{1}$ and ZhaoMin Cao ${ }^{1}$ \\ ${ }^{1}$ Key Lab of Education Ministry for Power Machinery and Engineering, School of Mechanical Engineering, \\ Shanghai Jiao Tong University, 800 Dongchuan Road, Shanghai 200240, China \\ ${ }^{2}$ Maritime College, Chongqing Jiaotong University, 66 Xuefu Road, Chongqing 40074, China
}

Correspondence should be addressed to Min Zhang; zhangmin@cqjtu.edu.cn

Received 19 June 2014; Accepted 6 November 2014; Published 20 November 2014

Academic Editor: Zhen-Lai Han

Copyright (C) 2014 Min Zhang et al. This is an open access article distributed under the Creative Commons Attribution License, which permits unrestricted use, distribution, and reproduction in any medium, provided the original work is properly cited.

\begin{abstract}
A concept of energy harvesting from vortex-induced vibrations of a rigid circular cylinder with two piezoelectric beams attached is investigated. The variations of the power levels with the free stream velocity are determined. A mathematical approach including the coupled cylinder motion and harvested voltage is presented. The effects of the load resistance, piezoelectric materials, and circuit combined on the natural frequency and damping of the vibratory system are determined by performing a linear analysis. The dynamic response of the cylinder and harvested energy are investigated. The results show that the harvested level in SS and SP\&PS modes is the same with different values of load resistance. For four different system parameters, the results show that the bigger size of cylinder with PZT beams can obtain the higher harvested power.
\end{abstract}

\section{Introduction}

Energy harvesting from vibrations, such as ambient and aeroelastic vibrations, has been proposed for powering electronic components. Many published literatures focused on utilizing these vibrations to either supply power for low power consumption devices, like microelectromechanical systems and actuators $[1,2]$, and wireless sensors and health monitoring $[3,4]$ or replace batteries that have a finite life span and require hard and expensive maintenance [5]. Nowadays, great efforts have been made to investigate the energy harvesting from mechanical vibrations induced by base excitation. Conversion of aeroelastic vibrations of wings to electrical power was investigated by Erturk et al. [6], De Marqui Jr. et al. [7, 8], and Abdelkefi et al. [9-12]. And a few investigations [13-15] focused on the conversion of vortexinduced vibrations of fluttering flags or microstructured piezobimorph to electrical power.

Vortex-induced vibrations (VIV) are a kind of self-excited vibration. When the vortex shedding frequency becomes close to the natural frequency of vibration of the bluff body, a lock-in or synchronization phenomenon will happen [1622]. Williamson and coworkers [23-29] indicated that there are different response modes which depend on the massdamping parameter. Govardhan and Williamson [30] defined the "modified Griffin plot" to reveal the effect of Reynolds number for the VIV peak-amplitude response. Morse and Williamson [31-33] studied the vortex-induced vibrations prediction by employing controlled motion.

A literature survey shows that few efforts have been taken to study the VIV energy harvester in the inland rivers. But we all know that the flow direction of the inland rivers is always the same during a whole year, and the range of flow speeds is certain; for example, the flow speed is about $0.5-2 \mathrm{~m} / \mathrm{s}$ in the three-gorge reservoir area of the Yangtze River. The objective of this work is to investigate the possibility of using VIV oscillations of structures to harvest electrical power in the inland river. Particularly, we hope to decide the power levels that can be generated from VIV oscillations of a circular cylinder and variations of these levels with the free stream velocity. To this end, we attach two piezoelectric beams to the transverse displacement degree of freedom of the cylinder 
and develop a method for the coupled VIV-energy harvesting system. Because these vibrations and energy harvesting are nonlinear, so we also investigate the impacts of cylinder size, piezoelectric materials, load resistance, and circuit combined modes on the harvested power levels.

In this work, a mathematical approach is employed to investigate and analyze the problem of energy harvesting from VIV oscillations. Section 2 presents the mathematical model and the parameters of different combined modes. The impact of the load resistance on the natural frequency and damping of the oscillatory system is discussed through the linear analysis in Section 3. The discussion of the level of the power that can be harvested and the effects of the piezoelectric materials, load resistance, and circuit combined modes on the harvested power, voltage output, and displacement amplitude goes to Section 4. Conclusions are included in Section 5 .

\section{Mathematical Model}

As shown in Figure 1, two piezoelectric beams are attached to the transverse displacement of the cylinder to harvest energy. The governing equation of motion of a spring-mounted rigid cylinder and the Gauss law $[6,9]$ are coupled as

$$
\begin{gathered}
M \ddot{Y}+2 M \xi \omega_{n} \dot{Y}+M \omega_{n}^{2} Y-\theta V=F(t), \\
C \dot{V}+\frac{V}{R}+\theta \dot{Y}=0,
\end{gathered}
$$

where $M$ is the oscillating mass, $\xi$ is the damping ratio, $\omega_{n}$ is the natural frequency in water, and $F(t)$ is the fluid force in the transverse direction. $\theta$ is the effective electromechanical coupling coefficient and $V$ is the harvested voltage across the load resistance. $R$ is the equivalent load resistance and $C$ is the equivalent capacitance of the piezoelectric layer. The piezoelectric layers of the bimorph cantilever can be combined in series or parallel [34], and there are two bimorph cantilevers existing in our system, so there will be four circuit combined modes, as shown in Figure 2.

(1) SS mode: the piezoelectric layers of one cantilever are combined in series and the two cantilevers are combined in series.

(2) SP mode: the piezoelectric layers of one cantilever are combined in series and the two cantilevers are combined in parallel.

(3) PS mode: the piezoelectric layers of one cantilever are combined in parallel and the two cantilevers are combined in series.

(4) PP mode: the piezoelectric layers of one cantilever are combined in parallel and the two cantilevers are combined in parallel.

The equivalent circuits for a load resistance of these modes are shown in Figure 3. The equivalent capacitance $C$, the effective electromechanical coupling coefficient $\theta$, and the equivalent load resistance $R$ of these four modes can be written as follows.

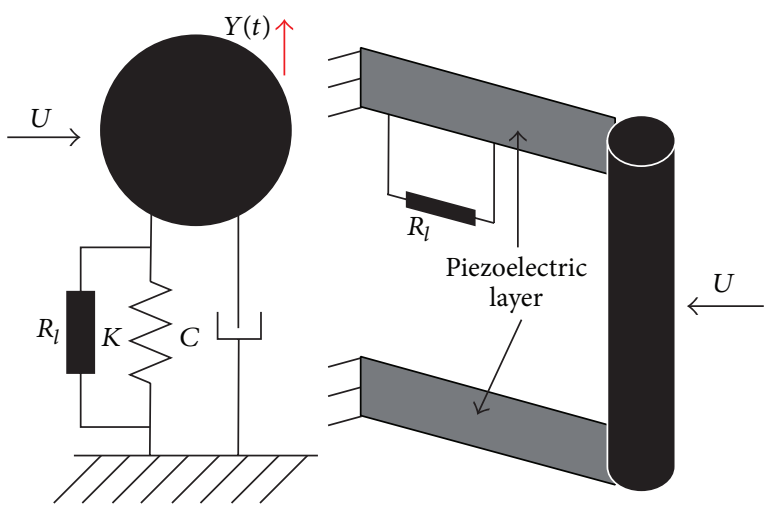

FIGURE 1: Schematic of a piezoelectric energy harvester of a freely oscillating cylinder.

For the SS mode,

$$
\begin{gathered}
C=\frac{C_{p}}{4}=\frac{\varepsilon_{0} \varepsilon_{r} b L}{4 h_{p}}, \\
\theta=\theta_{p}=\frac{e_{31}\left(h_{p}+h_{s}\right) b}{2}, \\
R=R_{l} .
\end{gathered}
$$

For the SP\&PS mode,

$$
\begin{gathered}
C=C_{p}=\frac{\varepsilon_{0} \varepsilon_{r} b L}{h_{p}}, \\
\theta=2 \theta_{p}=e_{31}\left(h_{p}+h_{s}\right) b, \\
R=R_{l} .
\end{gathered}
$$

For the PP mode,

$$
\begin{gathered}
C=C_{p}=\frac{\varepsilon_{0} \varepsilon_{r} b L}{h_{p}}, \\
\theta=\theta_{p}=\frac{e_{31}\left(h_{p}+h_{s}\right) b}{2}, \\
R=4 R_{l},
\end{gathered}
$$

where $C_{p}$ is the capacitance of a single piezoelectric layer, $\theta_{p}$ is the electromechanical coupling coefficient, $R_{l}$ is the load resistance, $e_{31}$ is the piezoelectric constant, $\varepsilon_{0}$ is the vacuum permittivity, $\varepsilon_{r}$ is the relative permittivity, $h_{p}, b$, and $L$ are used to represent the height, width, and length of the piezoelectric layer, and $h_{s}$ is the height of the substructure.

\section{Linear Analysis of the Electromechanical Model}

In order to determine the effects of the load resistance and circuit combined modes on the structural natural frequency and damping of the electromechanical system, we perform 


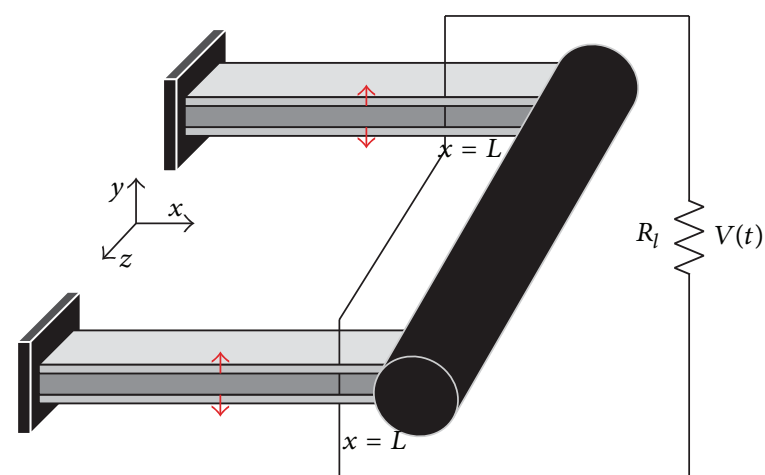

(a)

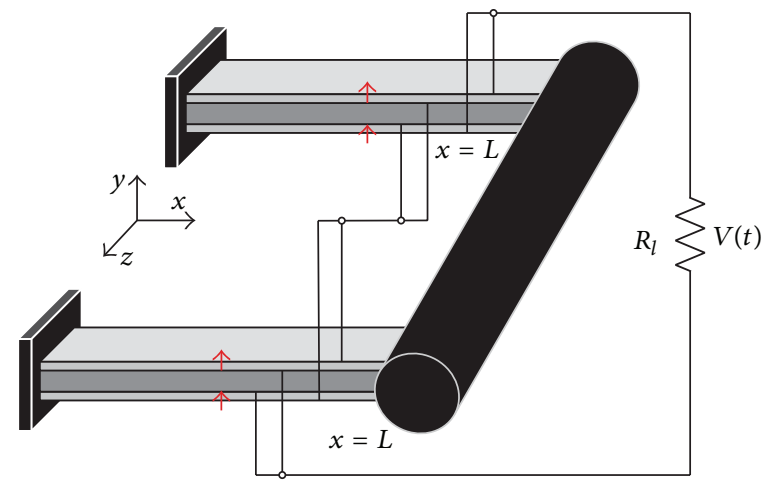

Piezoelectric layer Substructure $\begin{array}{ll}\uparrow & \text { Substructure } \\ & \text { Poling direction }\end{array}$

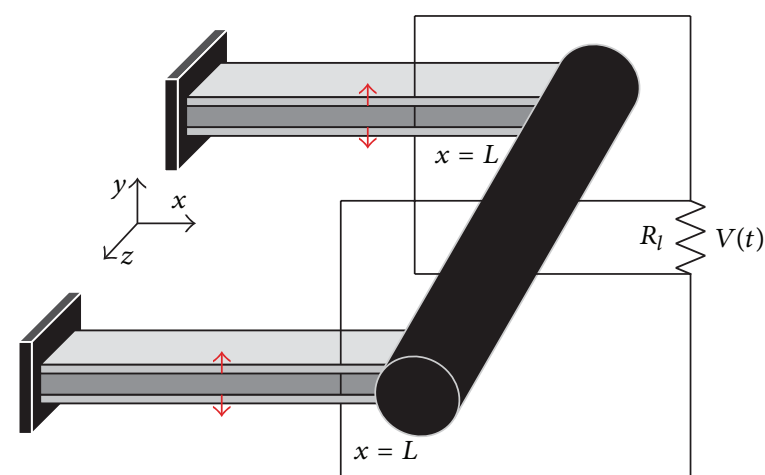

(b)

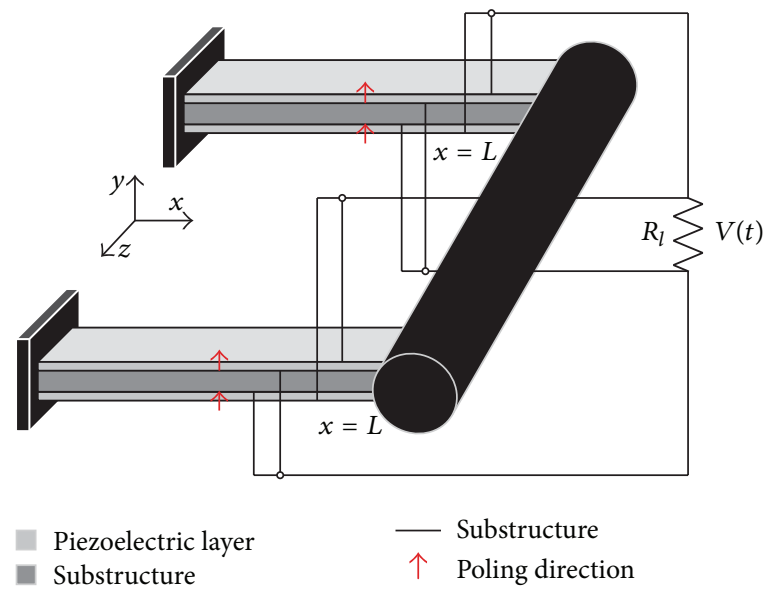

(d)

(c)

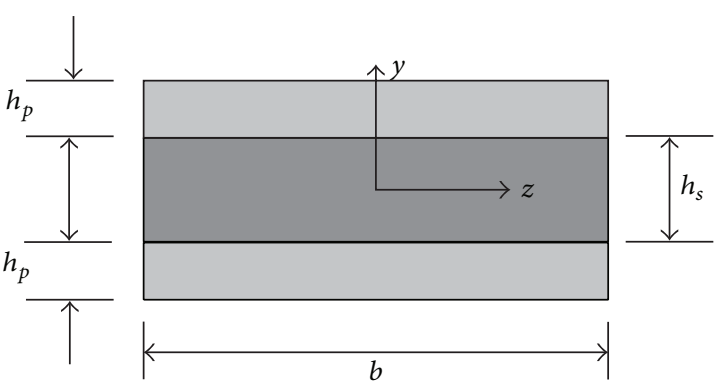

(e)

FIGURE 2: Energy harvesting systems with (a) SS mode, (b) SP mode, (c) PS mode, and (d) PP mode and (e) cross-sectional view of a bimorph cantilever.

a linear analysis of the governing equation of the cylinder and the Gauss law equation. We use the following state variables.

We rewrite the equations of the motion as

$$
\begin{gathered}
\dot{X}_{1}=X_{2}, \\
\dot{X}_{2}=-2 \xi \omega_{n} X_{2}-\omega_{n}^{2} X_{1}+\frac{\theta}{M} X_{3}, \\
\dot{X}_{3}=-\frac{1}{R C} X_{3}-\frac{\theta}{C} X_{2},
\end{gathered}
$$

where $X_{1}=Y, X_{2}=\dot{Y}$, and $X_{3}=V$.
These equations can be expressed in the following matrix form:

$$
\dot{\mathbf{X}}=B \mathbf{X}
$$

$$
B=\left[\begin{array}{ccc}
0 & 1 & 0 \\
-\omega_{n}^{2} & -2 \xi \omega_{n} & \frac{\theta}{M} \\
0 & -\frac{\theta}{C} & -\frac{1}{R C}
\end{array}\right] \text {, }
$$

where $B$ includes all parameters that affect the linear part of the system. This matrix can be used to investigate the effects 


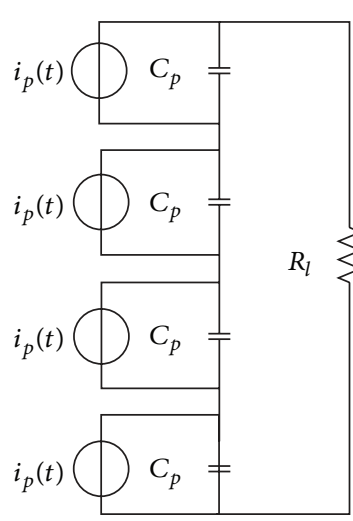

(a)

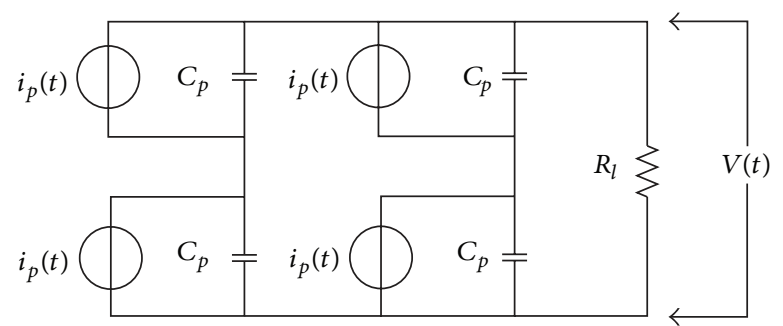

(b)

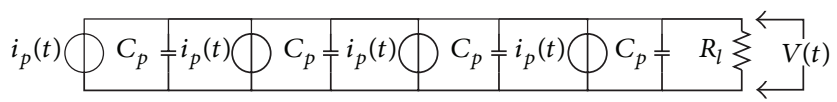

(c)

FIgURE 3: The equivalent circuits of (a) SS mode, (b) SP\&PS mode, and (c) PP mode.

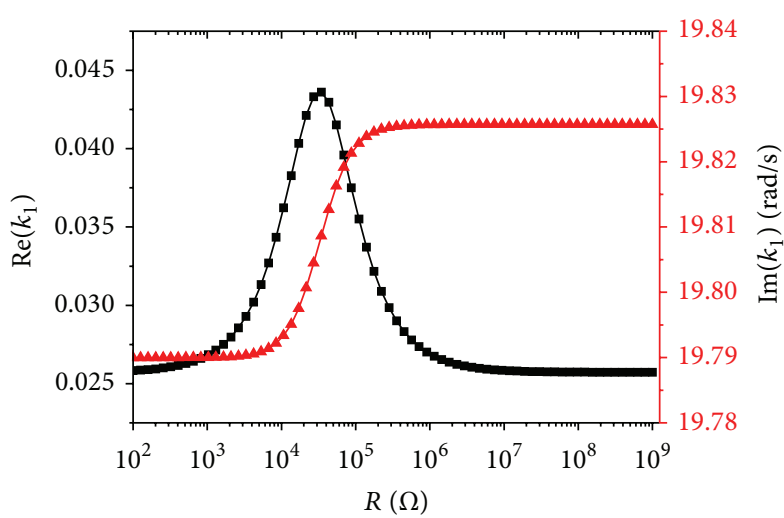

(a)

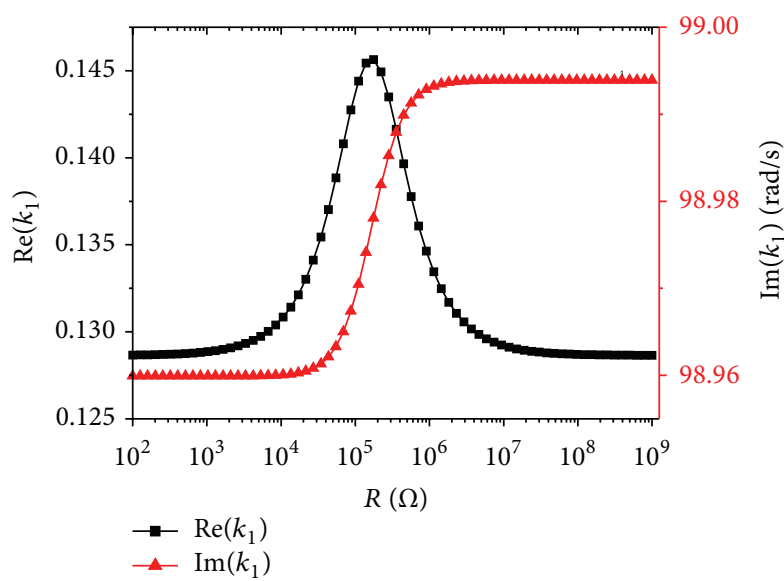

(c)

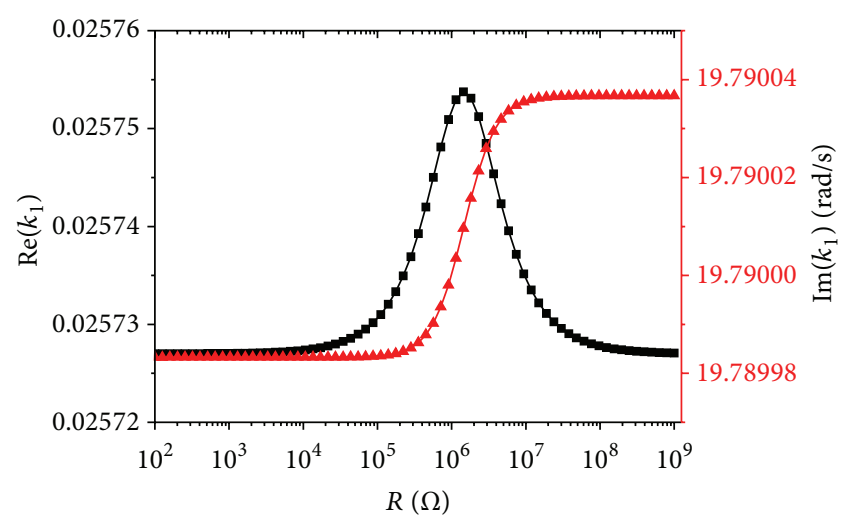

(b)

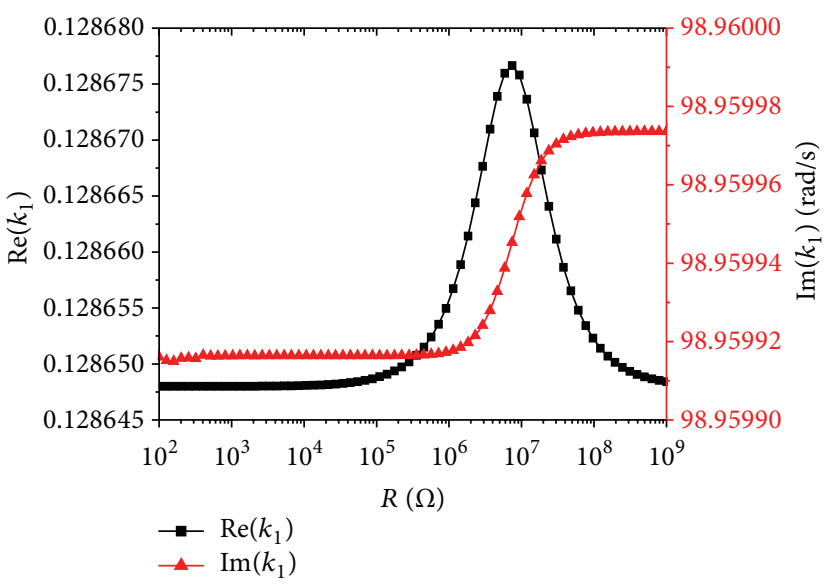

(d)

FIGURE 4: Variations of the imaginary parts and the real parts of the electromechanical model with the load resistance for (a) System 1, (b) System 2, (c) System 3, and (d) System 4 in SP\&PS mode. 
TABle 1: Parameters of the four considered configurations.

\begin{tabular}{|c|c|c|c|c|c|}
\hline Parameter & Description & System 1 & System 2 & System 3 & System 4 \\
\hline$M$ & Oscillating mass (kg) & 2.968 & 2.953 & 0.02477 & 0.02378 \\
\hline$D$ & Diameter $(\mathrm{m})$ & 0.05 & 0.05 & 0.01 & 0.01 \\
\hline$\xi$ & Damping ratio & 0.0013 & 0.0013 & 0.0013 & 0.0013 \\
\hline$\omega_{n}$ & Natural frequency (rad/s) & 19.79 & 19.79 & 98.96 & 98.96 \\
\hline$\theta_{p}$ & Electromechanical coupling coefficient & 0.002537 & $1.376 e-5$ & $1.011 e-4$ & $6.099 e-7$ \\
\hline$C_{p}$ & Capacitance (F) & $1.532 e-6$ & $3.453 e-8$ & $6.130 e-8$ & $1.381 e-9$ \\
\hline$m^{*}$ & Mass ratio & 1.535 & 1.507 & 1.629 & 1.523 \\
\hline
\end{tabular}

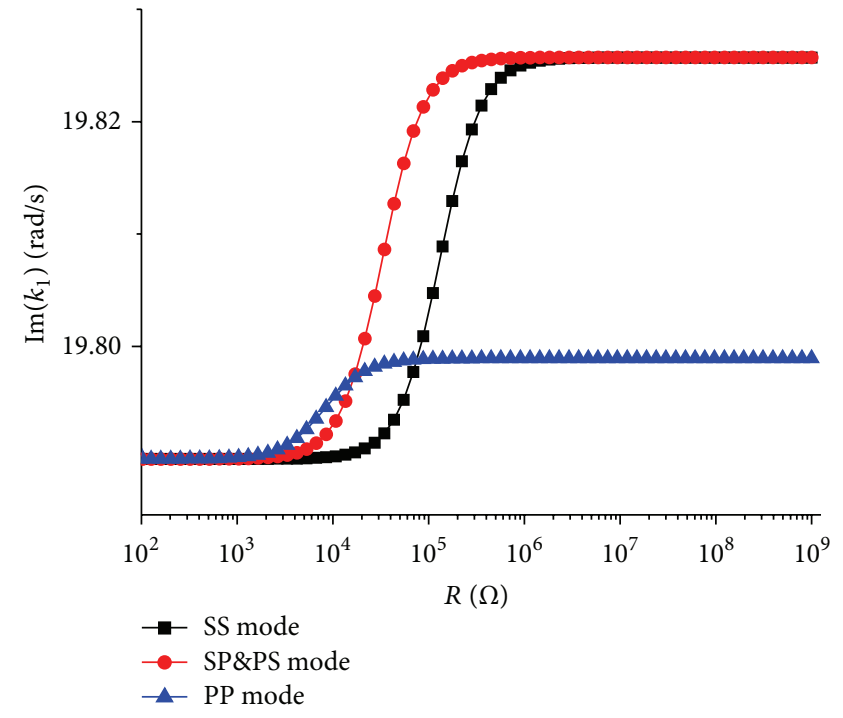

(a)

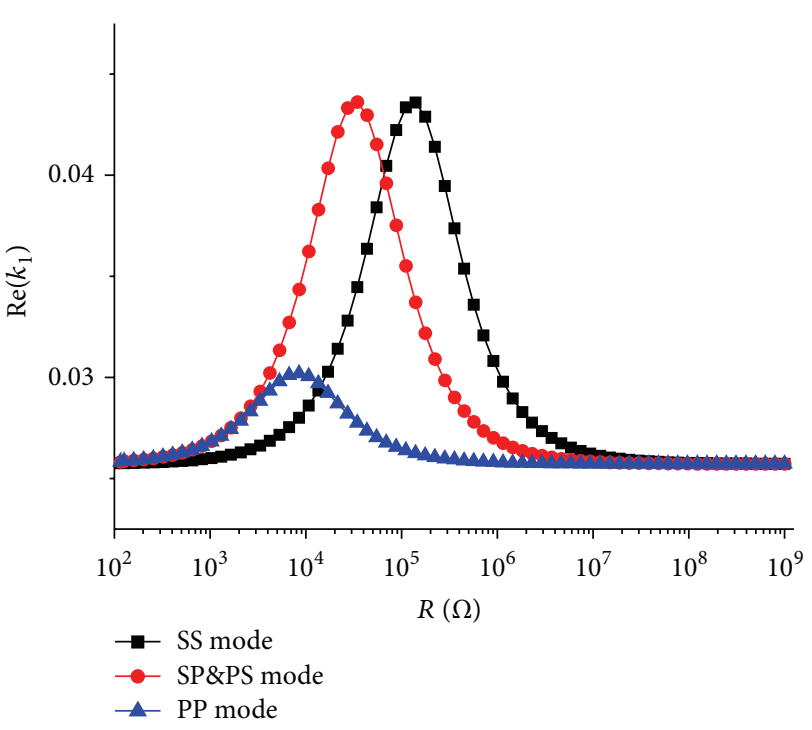

(b)

FIGURE 5: Variations of (a) the imaginary parts and (b) the real parts of the electromechanical model with the load resistance for System 1 in SS, SP\&PS, and PP modes.

of load resistance, damping ratio, and electromechanical coupling coefficients on the structural natural frequency and damping of electromechanical system. The matrix $B$ in (8) has three eigenvalues $k_{i}, i=1,2,3$. Abdelkefi et al. [35, 36] indicated that the first two eigenvalues are similar to those of a pure vibration system without piezoelectricity effect. The real part of these eigenvalues represents the damping coefficient and the positive imaginary part corresponds to the global frequency of the coupled system. The third eigenvalue, which is due to the electromechanical coupling, is always negative. Then the imaginary and real parts of $k_{1}$ can be expressed as

$$
\begin{gathered}
\operatorname{Im}\left(k_{1}\right)=\omega_{\mathrm{em}} \sqrt{1-\xi_{\mathrm{em}}^{2}}, \\
\operatorname{Re}\left(k_{1}\right)=\omega_{\mathrm{em}} \xi_{\mathrm{em}},
\end{gathered}
$$

where $\omega_{\mathrm{em}}$ and $\xi_{\mathrm{em}}$ are used to represent the natural frequency and damping ratio of the electromechanical system.

In this work, we consider four different configurations, namely, System 1, System 2, System 3, and System 4. The piezoelectric materials of System 1 and System 3 are PZT, while the piezoelectric materials of System 2 and System 4 are
PVDF. Firstly, we compare these four systems in SP\&PS mode to neglect the effects of the circuit connections mode, and, then, in order to analyze the effects of the circuit connections mode, we make the linear analysis of System 1 in SS, SP\&PS, and PP modes. The parameters of these four configurations are presented in Table 1.

Figure 4 shows variations of the imaginary and real parts of the complex eigenvalues with the load resistance. In the four systems, a steep increase of the imaginary part is noted over the load resistance values. For System 1, the steep increase occurs between $10^{4} \Omega$ and $6 \times 10^{5} \Omega$; for System 2 , the steep increase occurs between $3 \times 10^{5} \Omega$ and $10^{7} \Omega$; for System 3, the steep increase occurs between $4 \times 10^{4} \Omega$ and $10^{6} \Omega$; for System 4, the steep increase occurs between $10^{6} \Omega$ and $10^{8} \Omega$. From the imaginary part of this eigenvalue, we can obtain the global frequency of these four systems. For System 1, the global frequency is approximately equal to $19.790 \mathrm{rad} / \mathrm{s}$ between $10^{2} \Omega$ and $10^{3} \Omega$. Increasing the load resistance causes an increase in the global frequency with a steep increase to values near $19.826 \mathrm{rad} / \mathrm{s}$ when the load resistance is near $10^{6} \Omega$. For System 2, the global frequency 


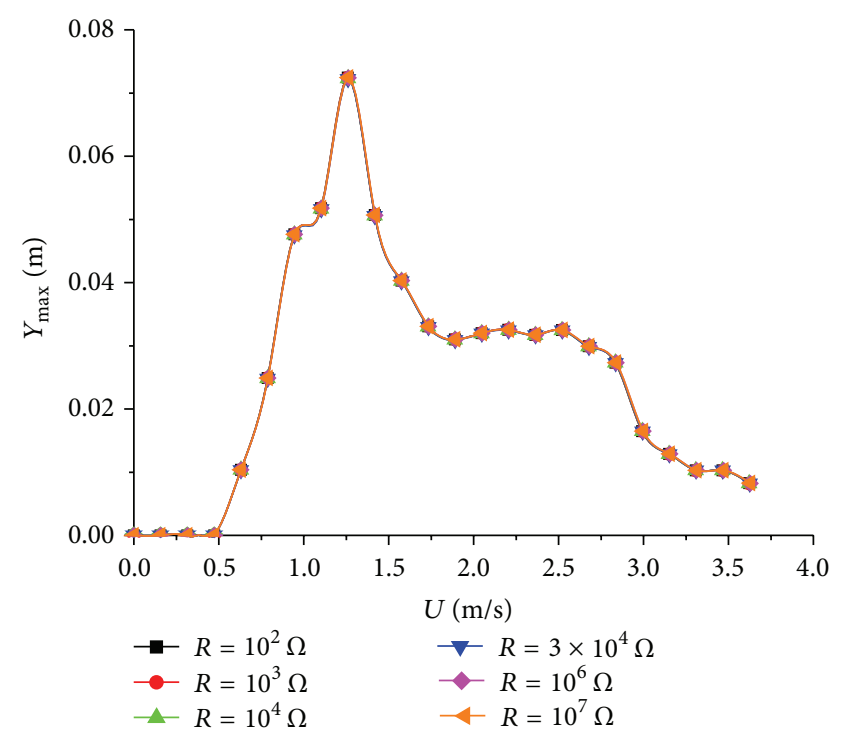

(a)

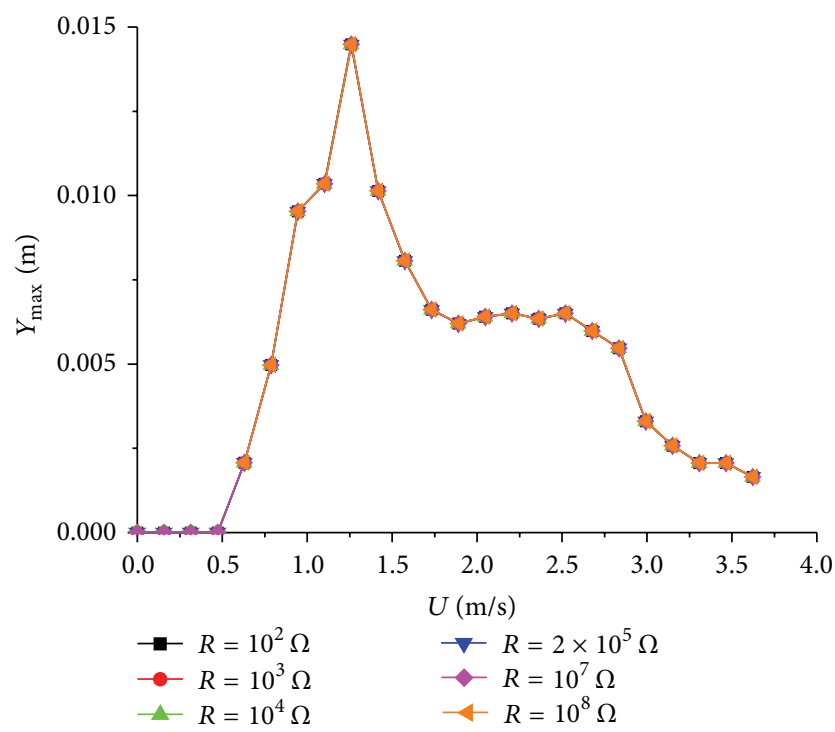

(c)

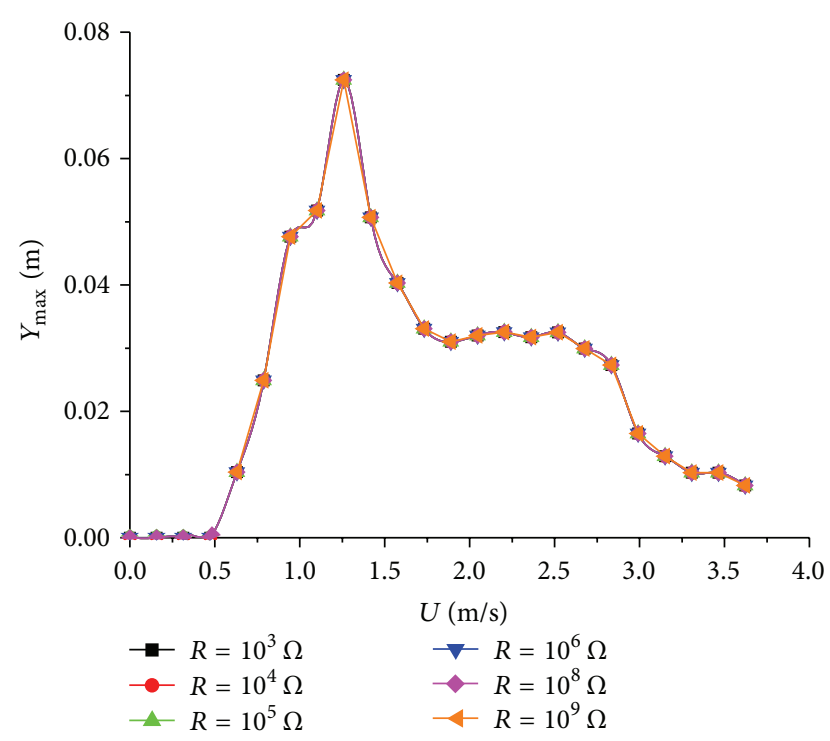

(b)

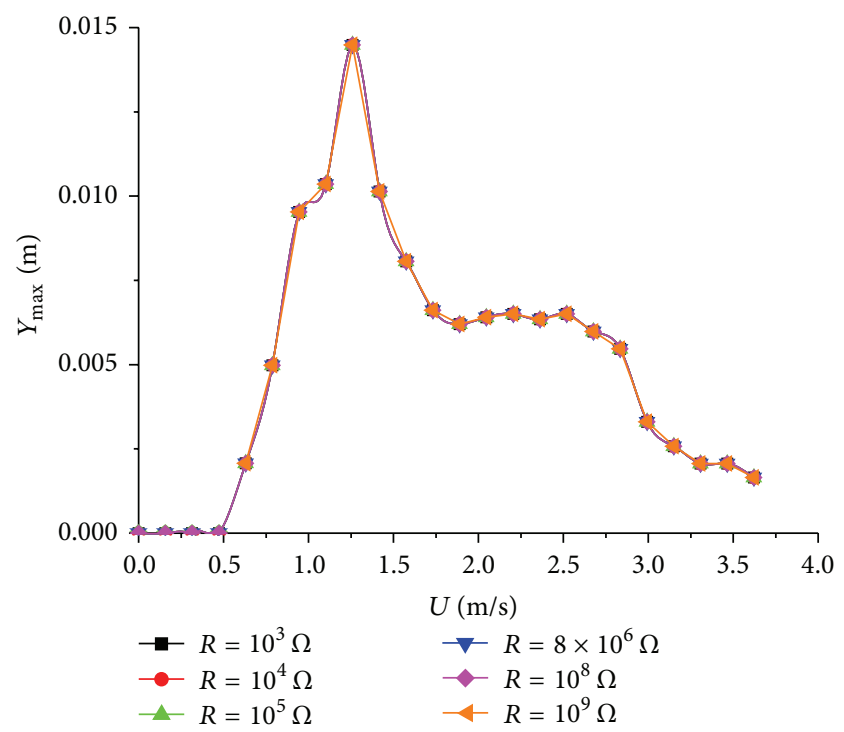

(d)

FiguRE 6: Frequency-response curves of the transverse displacement for different values of the load resistance and for (a) System 1, (b) System 2, (c) System 3, and (d) System 4.

is approximately equal to $19.790 \mathrm{rad} / \mathrm{s}$ between $10^{2} \Omega$ and $10^{4} \Omega$. Increasing the load resistance causes an increase in the global frequency with a steep increase to values near $19.790 \mathrm{rad} / \mathrm{s}$ when the load resistance is near $10^{7} \Omega$. For System 3, the global frequency is approximately equal to $98.960 \mathrm{rad} / \mathrm{s}$ between $10^{2} \Omega$ and $10^{4} \Omega$. Increasing the load resistance causes an increase in the global frequency with a steep increase to values near $98.994 \mathrm{rad} / \mathrm{s}$ when the load resistance is near $3 \times 10^{6} \Omega$. For System 4 , the global frequency is approximately equal to $98.960 \mathrm{rad} / \mathrm{s}$ between $10^{3} \Omega$ and $10^{5} \Omega$. Increasing the load resistance causes an increase in the global frequency with a steep increase to values near $98.960 \mathrm{rad} / \mathrm{s}$ when the load resistance is near $10^{8} \Omega$.
From Figure 4 we can also note that the real part which indicates the electromechanical damping reaches maxima at a specific value of the load resistance for the four systems.

This linear analysis gives a clear idea about the effect of the load resistance and piezoelectric material on the global frequency and the electromechanical damping. We note that although there is a steep increase of the imaginary part in the four systems, the absolute value changes of the global frequency are not significant, especially for System 2 and System 4 with PVDF piezoelectric material. The value changes of the electromechanical damping are also small, particularly for System 2 and System 4.

Figure 5 shows variations of the imaginary and real parts of the complex eigenvalues with the load resistance for 


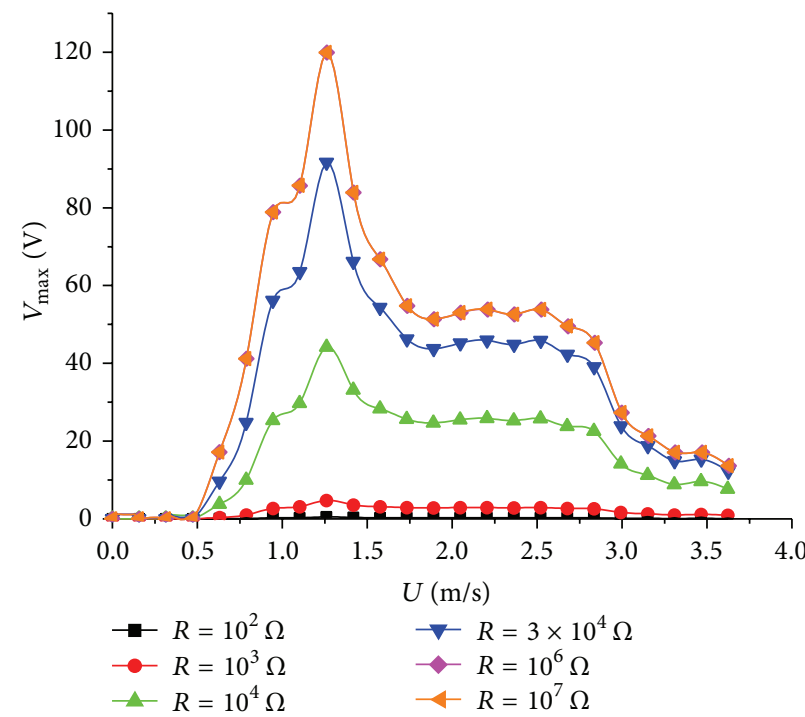

(a)

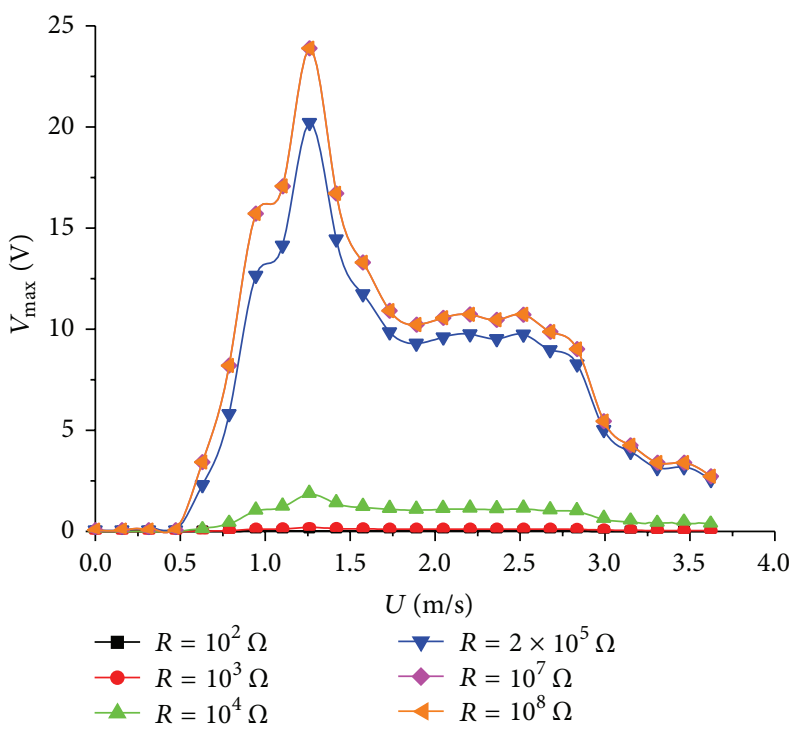

(c)

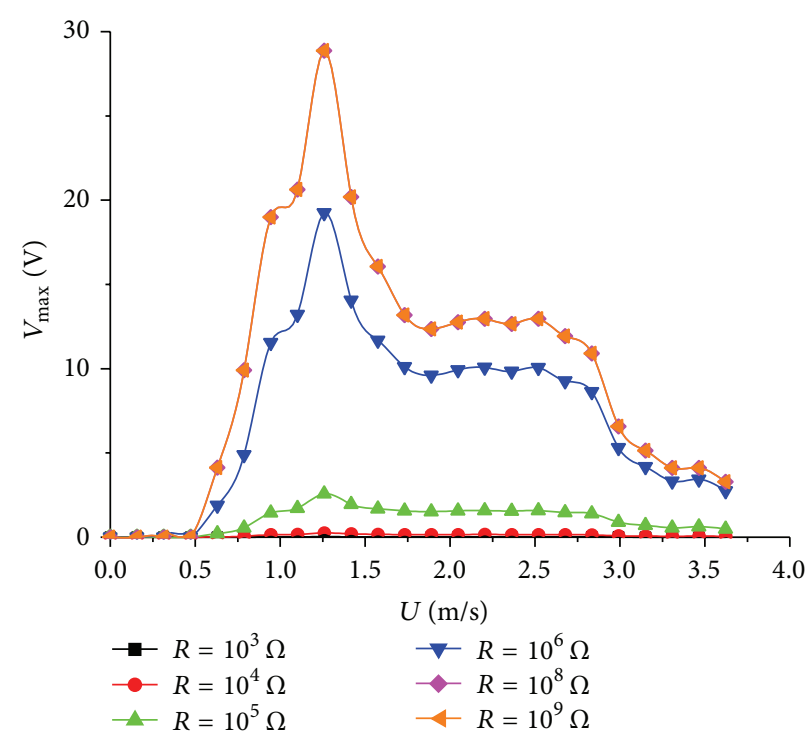

(b)

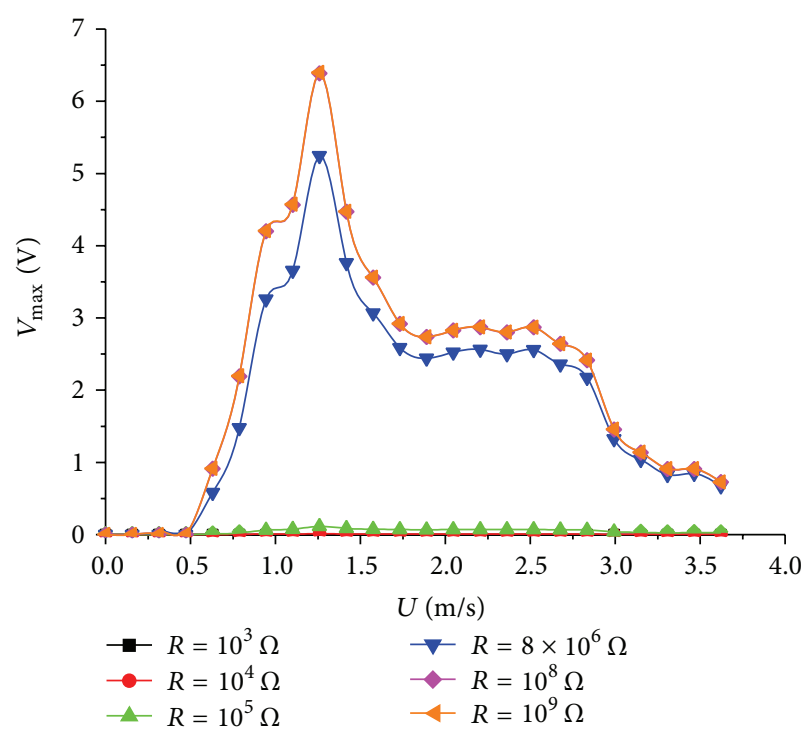

(d)

FIGURE 7: Frequency-response curves of the harvested voltage for different values of the load resistance and for (a) System 1, (b) System 2, (c) System 3, and (d) System 4.

System 1 in different combined modes. From Figure 5(a) we note that the global frequency change is smallest in PP mode, and the global frequency changes in SS mode and SP\&PS mode are almost the same. We also note that the global frequency increase regions are different. The PP mode is between $3 \times 10^{3} \Omega$ and $10^{5} \Omega$, the SP\&PS mode is between $10^{4} \Omega$ and $6 \times 10^{5} \Omega$, and the SS mode is between $2 \times 10^{4} \Omega$ and $5 \times 10^{6} \Omega$. From Figure $5(\mathrm{~b})$ we note that the maximum electromechanical damping is smallest in PP mode, the same value in SS mode and SP\&PS mode. We note also that the region of load resistance over which the electromechanical damping is relatively high coincides with the region over which the steep increase in the global frequency occurs.

\section{Nonlinear Analysis of the Electromechanical System}

The dynamics of a freely vibrating cylinder from controlled vibration force measurement (for both steady-state and transient behaviors) is modeled by Morse and Williamson [33].

The equation of motion for vortex-induced vibration in the transverse $(y)$ direction (normal to the flow) is as follows:

$$
M \ddot{Y}+2 M \xi \omega_{n} \dot{Y}+M \omega_{n}^{2} Y=F(t) .
$$

If we substitute (9) into the equation of motion (10), we can obtain the fluid-electromechanical coupled equation:

$$
M \ddot{Y}+2 M \xi_{\mathrm{em}} \omega_{\mathrm{em}} \dot{Y}+M \omega_{\mathrm{em}}^{2} Y=F(t) .
$$




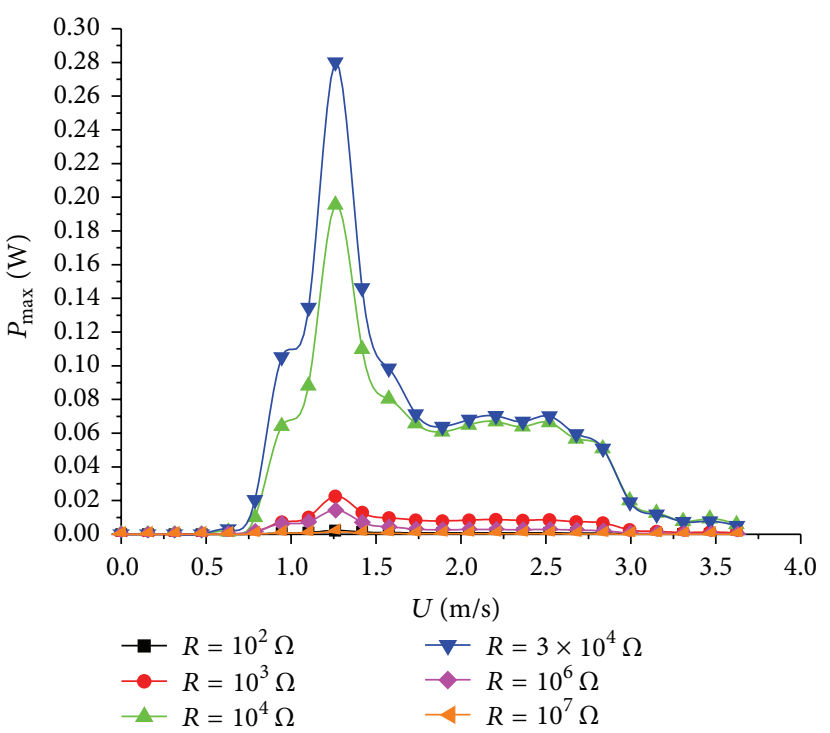

(a)

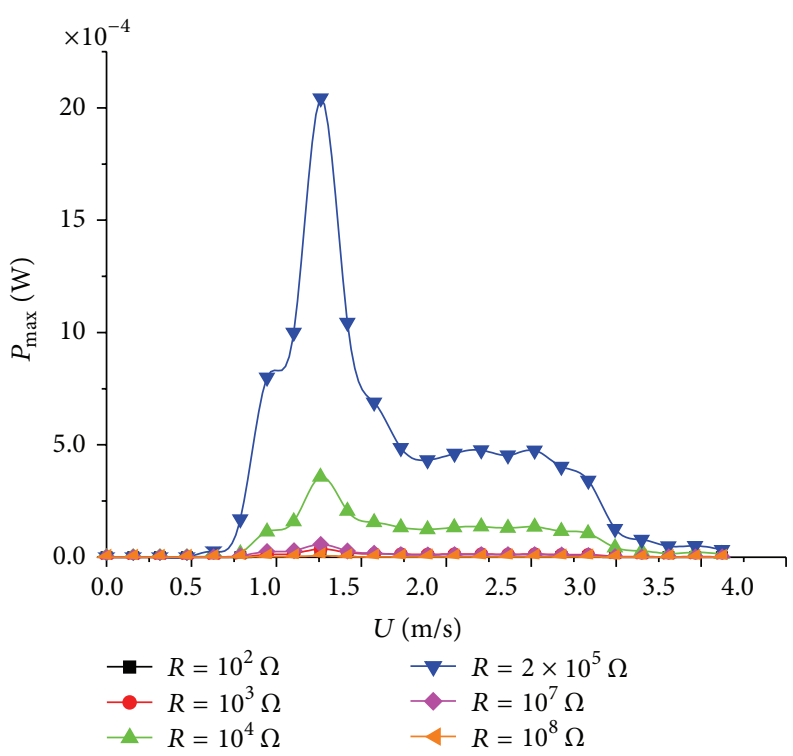

(c)

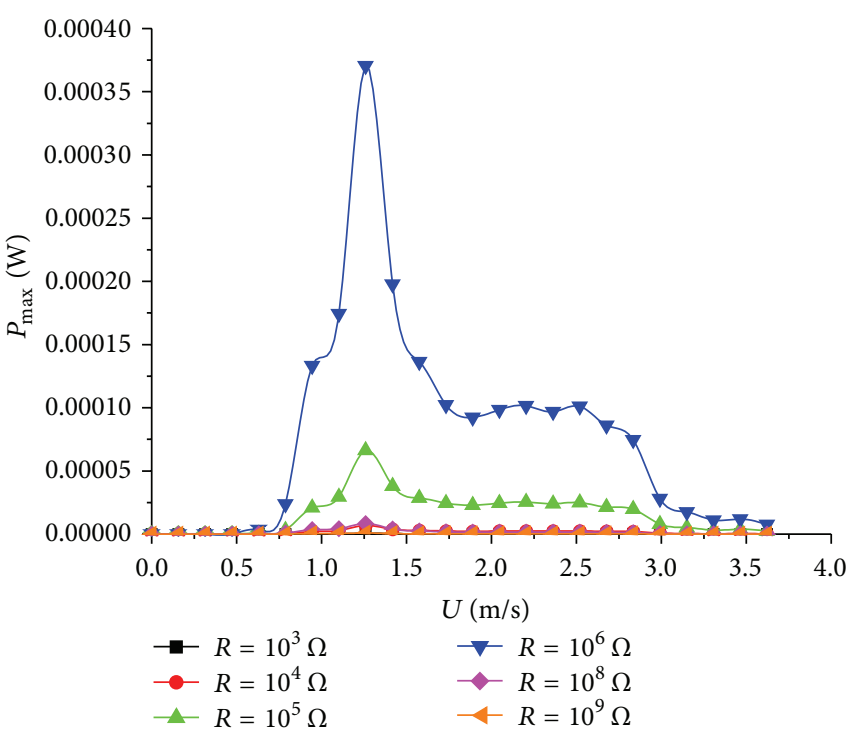

(b)

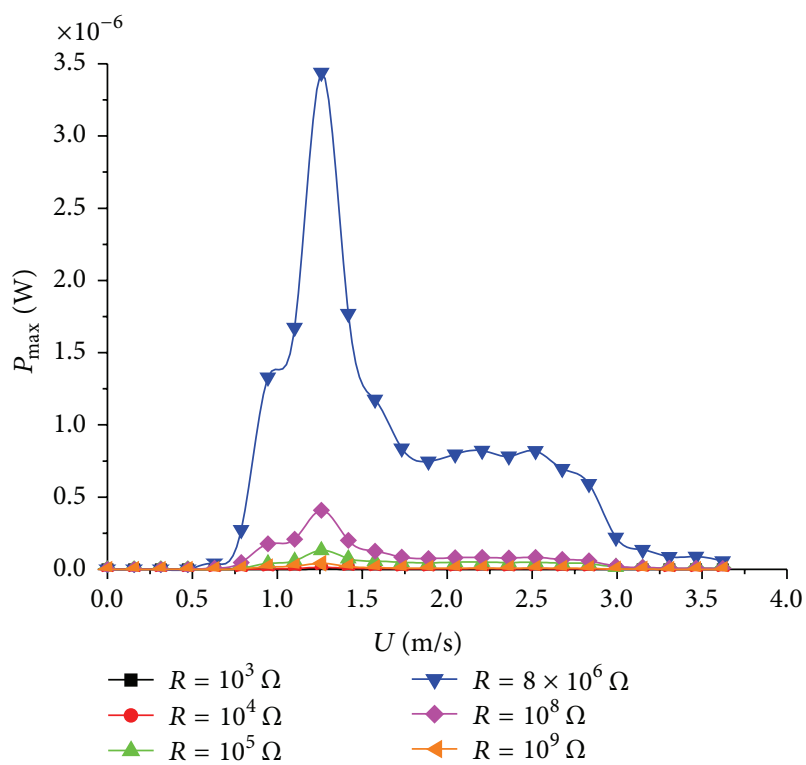

(d)

FIGURE 8: Frequency-response curves of the harvested power for different values of the load resistance and for (a) System 1, (b) System 2, (c) System 3, and (d) System 4.

4.1. Steady-State Equations of Motion for Free Vibration. We assume that the motion takes on a sinusoidal form with the periodic vortex-formation mode and the displacement and force equations can be expressed as

$$
\begin{gathered}
Y(t)=A \sin \omega t, \\
F(t)=F_{1} \sin (\omega t+\phi),
\end{gathered}
$$

where $\omega=2 \pi f$ is the oscillation frequency and $\phi$ is the phase angle between the fluid force and the body displacement.
We substitute (12) and (13) into the fluid-electromechanical coupled equation (11), and we can obtain the "amplitude equation" and the "frequency equation" as follows:

$$
\begin{gathered}
A^{*}=\frac{1}{4 \pi^{3}} \frac{C_{Y} \sin \phi}{\left(m^{*}+C_{A}\right) \xi_{\mathrm{em}}}\left(\frac{U^{*}}{f^{*}}\right)^{2} f^{*}, \\
f^{*}=\sqrt{\frac{m^{*}+C_{A}}{m^{*}+C_{E A}}},
\end{gathered}
$$

where $A^{*}=A / D, D$ is the cylinder diameter, $C_{Y} \sin \phi$ is the force coefficient in phase with the body velocity, $U^{*}=$ $U / f_{n} D, U$ is the free stream velocity, $f_{n}$ is the natural 


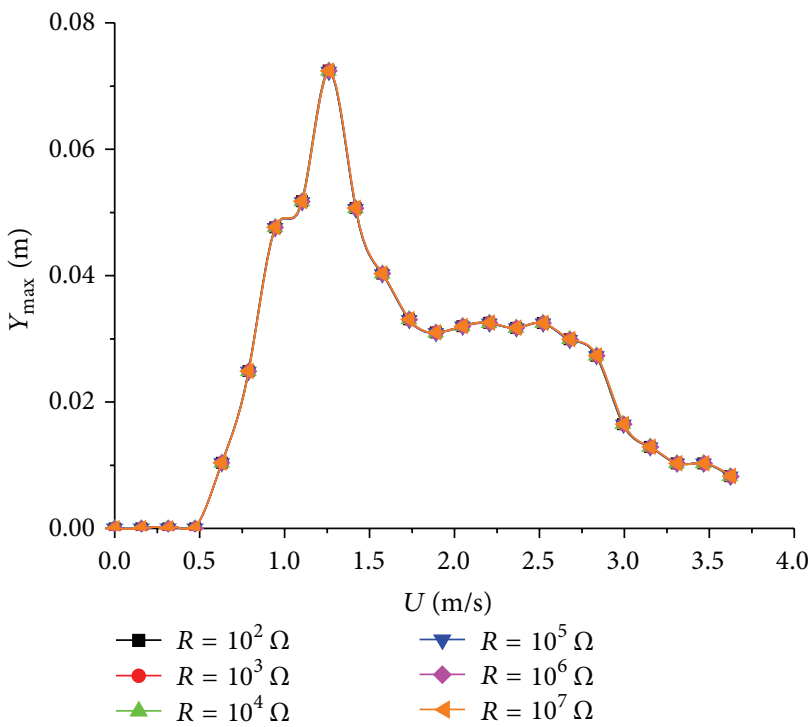

(a)

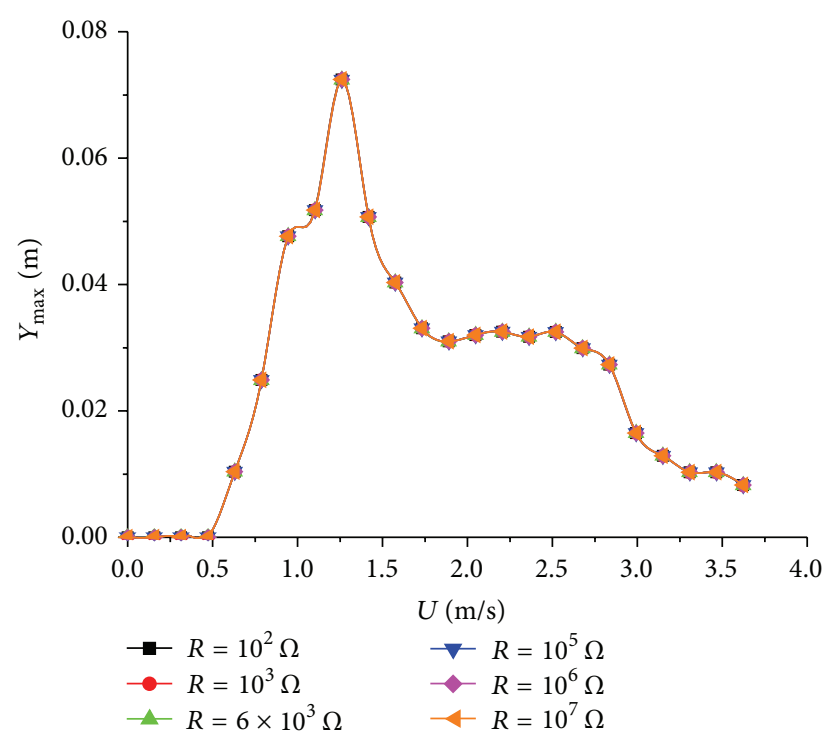

(b)

FIGURE 9: Frequency-response curves of the transverse displacement for different values of the load resistance and for System 1 in (a) SS mode and (b) PP mode.

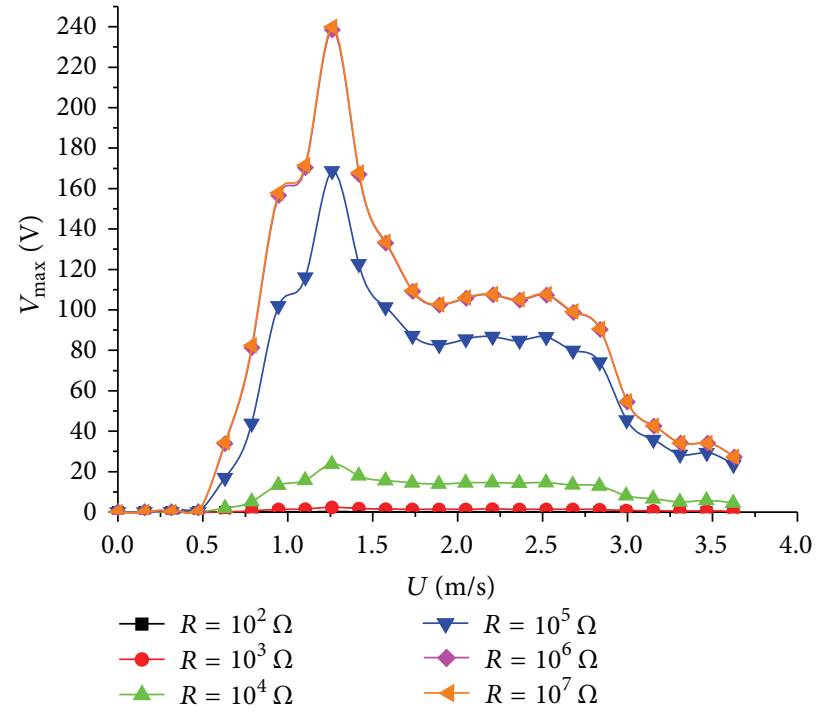

(a)

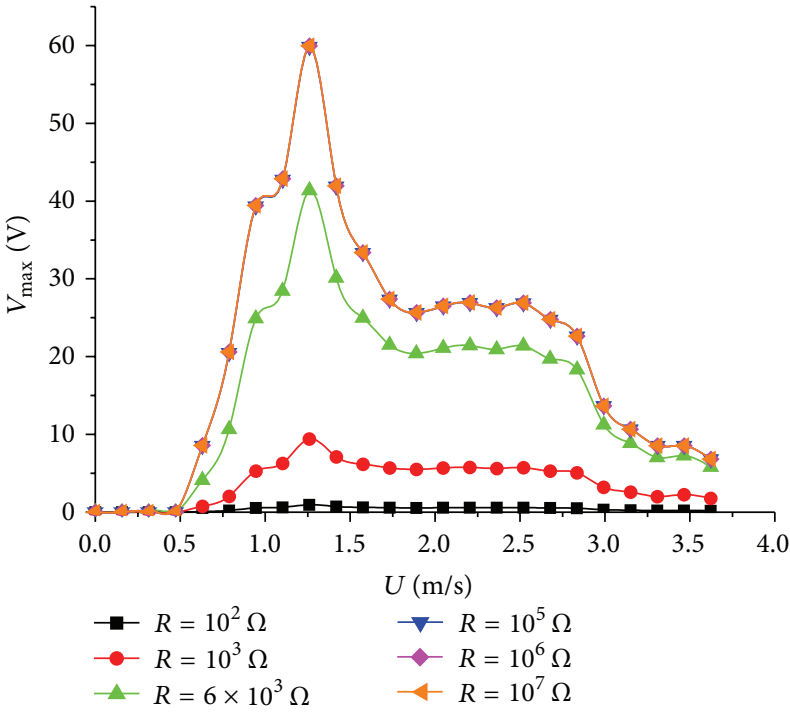

(b)

FIGURE 10: Frequency-response curves of the harvested voltage for different values of the load resistance and for System 1 in (a) SS mode and (b) PP mode.

frequency in water, $m^{*}=4 m / \pi \rho D^{2} L$ is the mass ratio, $\rho$ is the fluid density, $L$ is the submerged cylinder length, $C_{A}$ is the potential flow added-mass coefficient $\left(C_{A}=1.0\right.$ for a circular cylinder $), C_{E A}=\left(1 / 2 \pi^{3}\right)\left(C_{Y} \cos \phi / A^{*}\right)\left(U^{*} / f^{*}\right)^{2}$ is an "effective" added-mass coefficient, and $f^{*}=f / f_{n}$ is the frequency ratio.

From the force contours in Figure 3 presented by [33], we can predict the steady-state (sinusoidal) response (amplitude, $A^{*}$, and frequency, $f^{*}$ ) of the coupled system for a given set of system parameters $\left\{m^{*}, \xi_{\mathrm{em}}, U^{*}\right\}$.
4.2. Quasi-Steady Model for Free Vibration Response. We still assume that the motion takes on a sinusoidal form with varying amplitude and frequency.

We introduce the "effective damping" and "effective mass" as follows:

$$
\begin{gathered}
\xi_{\mathrm{eff}}=\xi_{\mathrm{em}}-\frac{1}{4 \pi^{3}} \frac{C_{Y} \sin \phi}{\left(m^{*}+C_{A}\right) A^{*}}\left(\frac{U^{*}}{f^{*}}\right)^{2} f^{*}, \\
m_{\mathrm{eff}}^{*}=m^{*}+C_{E A} .
\end{gathered}
$$




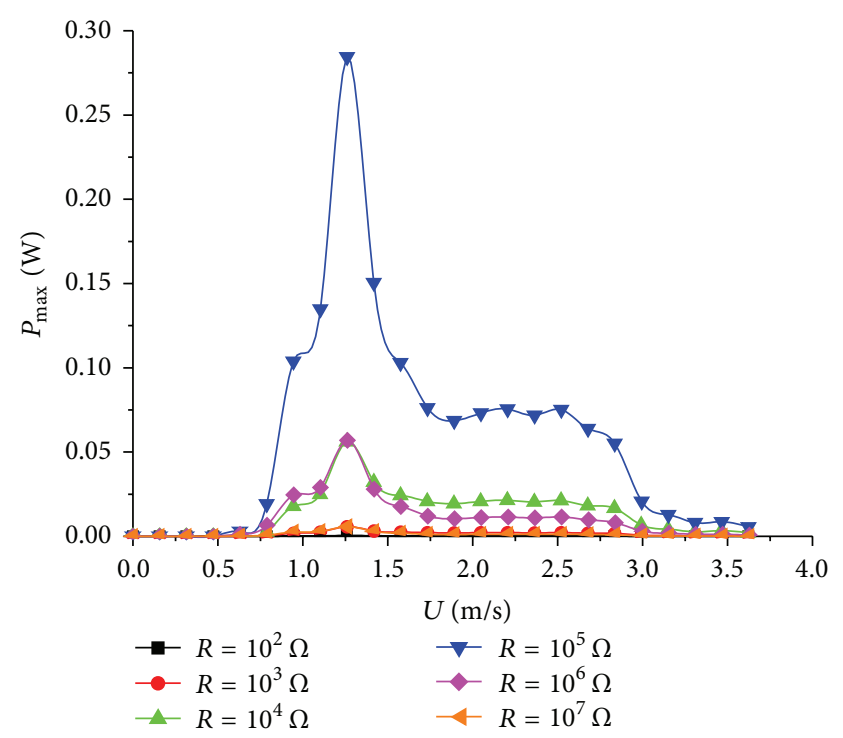

(a)

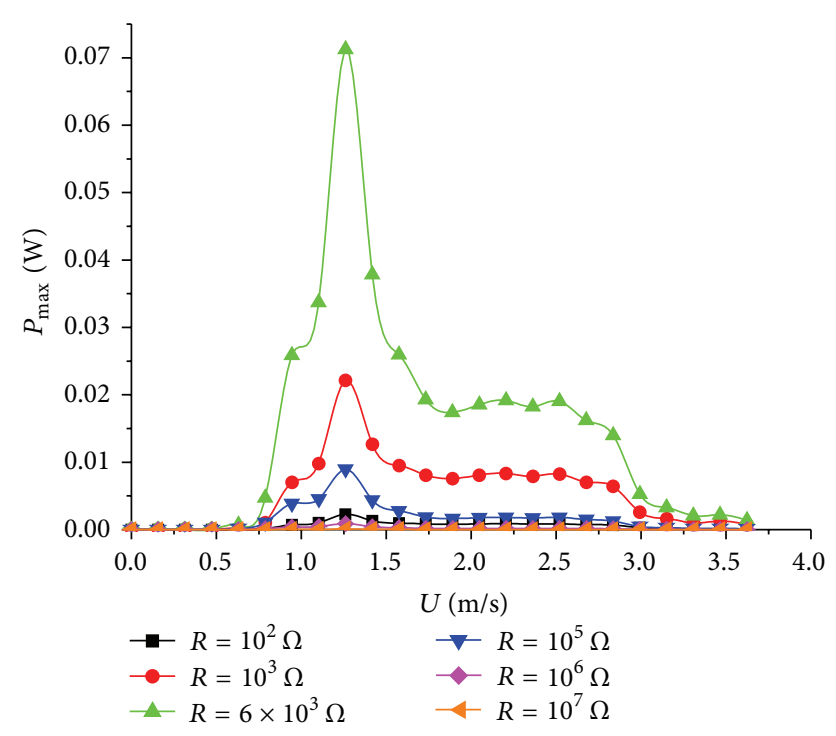

(b)

FIGURE 11: Frequency-response curves of the harvested power for different values of the load resistance and for System 1 in (a) SS mode and (b) PP mode.

The effective damping will determine if the amplitude increases or decreases as follows:

$$
\frac{d A^{*}}{d \tau}=\left(-2 \pi \xi_{\text {eff }} f^{*}\right) A^{*}
$$

The frequency ratio can be expressed as

$$
f^{*}=\sqrt{\frac{m^{*}+C_{A}}{m_{\mathrm{eff}}^{*}}} .
$$

Once we define the system parameters $\left\{m^{*}, \xi_{\text {em }}, U^{*}\right\}$ and initial values for $A^{*}$ and $f^{*}$, we can implement the quasisteady model.

We use the steady-state and quasi-steady model to calculate the dynamic response of the coupled system. More details about the steady-state and quasi-steady model can be found in Morse and Williamson [33].

4.3. The Harvested Voltage and Power. Once we obtain the coupled system dynamic response, we can substitute (12) into (2) to yield

$$
C \dot{V}+\frac{V}{R}+\theta A \omega \cos \omega t=0 .
$$

Solving (18), we can obtain

$$
V(t)=C_{1} e^{-t / R C}-\frac{A R \theta \omega(R C \omega \sin (\omega t)+\cos (\omega t))}{1+R^{2} C^{2} \omega^{2}} .
$$

The first term $C_{1} e^{-t / R C}$ on the right tends to 0 as the time $(t)$ increases, so we rewrite (19):

$$
V(t)=-\frac{A R \theta \omega}{\sqrt{1+R^{2} C^{2} \omega^{2}}} \sin (\omega t+\alpha),
$$

where $\cos \alpha=R C \omega / \sqrt{1+R^{2} C^{2} \omega^{2}}$ and $\sin \alpha=1 /$ $\sqrt{1+R^{2} C^{2} \omega^{2}}$.

From (20), we can obtain the maximum voltage $\left(V_{\max }\right)$ :

$$
V_{\max }=\frac{A R \theta \omega}{\sqrt{1+R^{2} C^{2} \omega^{2}}} .
$$

The maximum power $\left(P_{\max }\right)$ will be

$$
P_{\max }=\frac{V_{\max }^{2}}{R} .
$$

The dependence of the system response on the free stream velocity is shown in Figures 6 to 8. From Figure 6, we note that the synchronization regions of the four systems are among the same free stream velocities between $1.0 \mathrm{~m} / \mathrm{s}$ and $3.0 \mathrm{~m} / \mathrm{s}$. These phenomena are due to the small value changes of the global frequency and electromechanical damping, as aforementioned (Figure 4).

Variation of the maximum of the harvested voltage with the free stream velocity for different values of the load resistance is shown in Figure 7. We note that the maximum voltage near $120 \mathrm{~V}$ can be attainted for $R=10^{7} \Omega$ for System 1 , near $30 \mathrm{~V}$ for $R=10^{9} \Omega$ for System 2 , near $25 \mathrm{~V}$ for $R=10^{8} \Omega$ for System 3, and near $6.5 \mathrm{~V}$ for $R=10^{9} \Omega$ for System 4 , respectively. We also note that the maximum voltages for the four systems are obtained at the free stream velocity of $1.25 \mathrm{~m} / \mathrm{s}$.

Figure 8 shows the harvested power for different values of the load resistance for the four systems. They show maximum power levels near $0.28 \mathrm{~W}, 3.7 \times 10^{-4} \mathrm{~W}, 2 \times 10^{-3} \mathrm{~W}$, and $3.5 \times$ $10^{-6} \mathrm{~W}$ that can be attained for $R=3 \times 10^{4} \Omega, R=10^{6} \Omega$, $R=2 \times 10^{5} \Omega$, and $R=8 \times 10^{6} \Omega$ for System 1 , System 2 , System 3, and System 4 , respectively.

Figure 9 shows the transverse displacement for different values of the load resistance for System 1 in Figure 9(a) 


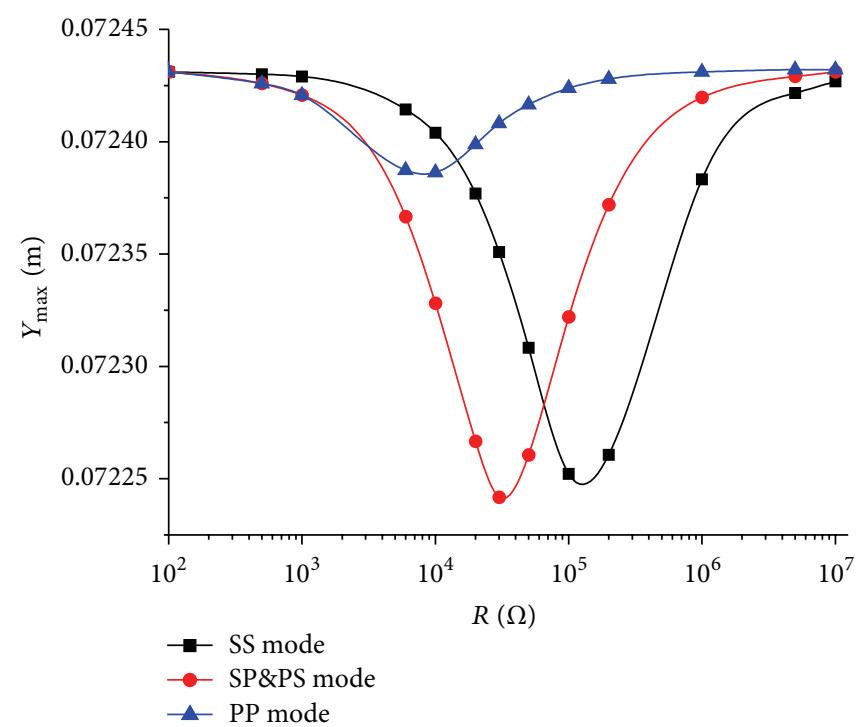

(a)

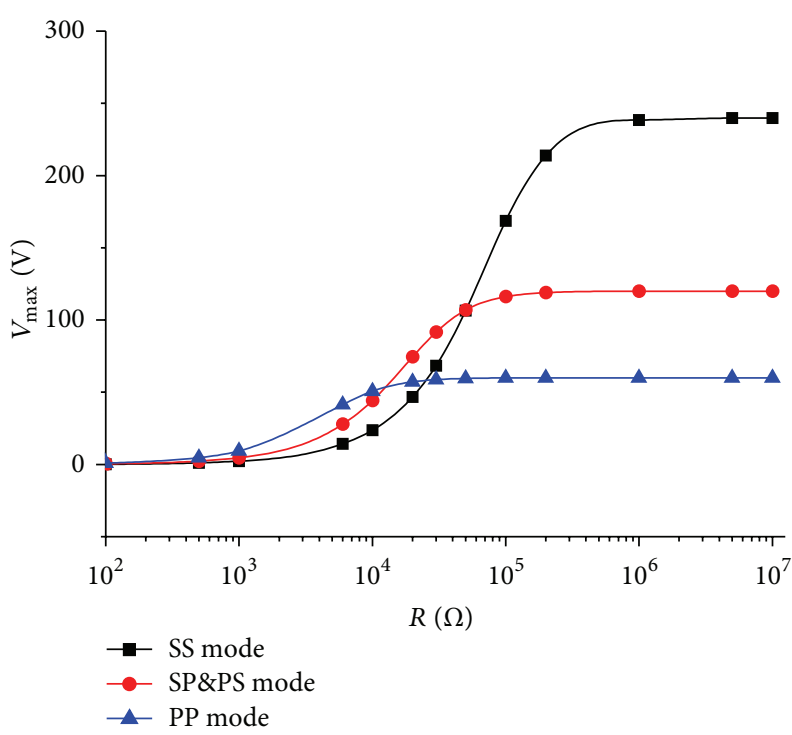

(b)

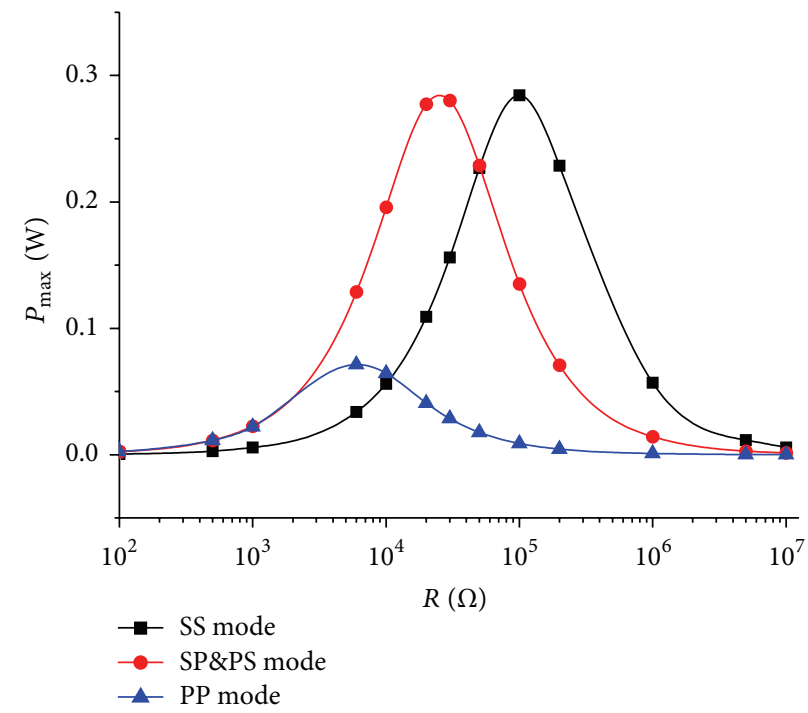

(c)

FIGURE 12: Variations of (a) the transverse displacement, (b) the harvested voltage, and (c) the harvested power with the load resistance for System 1 in SS, SP\&PS, and PP modes.

SS mode and Figure 9(b) PP mode. When we compared it with Figure 6(a), we note that the combined mode has no significant effect on the transverse displacement.

The harvested voltages for different values of the load resistance for System 1 in SS mode and PP mode are shown in Figures 10(a) and 10(b), respectively. We note that the maximum voltage near $240 \mathrm{~V}$ can be attained for $R=10^{7} \Omega$ in SS mode; meanwhile, the maximum voltage near $120 \mathrm{~V}$ and $60 \mathrm{~V}$ can be attained in SP mode (Figure 7(a)) and PP mode, respectively. We observed the effects of the harvested voltage which coursed by the combined mode. The harvested voltage is maximum in SS mode, then the second is SP mode, and the smallest is PP mode.
Figure 11 shows the harvested power for different values of the load resistance for System 1 in Figure 11(a) SS mode and Figure 11(b) PP mode. Compared with Figure 8(a), we note that the maximum power level near $0.28 \mathrm{~W}$ can be attained in both SS mode and SP mode, but for different load resistances. For SS mode, the corresponding load resistance is $R=10^{5} \Omega$ and for SP mode it is $R=3 \times 10^{4} \Omega$. Meanwhile, the maximum power level near $0.065 \mathrm{~W}$ can be attained for $R=10^{4} \Omega$ in PP mode. The maximum power and the optimal load resistance are affected by the combined mode, and the harvested power and the optimal load resistance are the smallest in PP mode.

Variations of the maximum of transverse displacement, the harvested voltage, and the harvested power with the load 
resistance for System 1 in SS, SP\&PS, and PP modes are shown in Figure 12. From Figures 12(a) and 12(c), we note that the transverse displacement is the smallest when the maximum harvested power is attained in all of the three combined modes. From Figure 12(b), we note that the harvested voltage increases as the load resistance is increased and reaches a constant value. The SS mode can obtain the largest harvested voltage, the harvested voltage of SP\&PS mode is medium, and the smallest voltage is attained in PP mode. And we also note that the maximum harvested power is attained before the voltage reaches the constant value in all of the three modes.

\section{Conclusions}

In the present study, the concept of energy harvesting from the vortex-induced vibration of a circular cylinder by attaching two piezoelectric beams was investigated. We developed a mathematical approach to solve the fluid-electromechanical coupled problem. The equivalent circuits of four different combined modes are presented. The dynamic response of the fluid-electromechanical coupled system is modeled by using the controlled vibration contour plots of fluid force. The results show that the piezoelectric materials, circuit combined mode, cylinder size, and load resistance influence the global natural frequency and the damping of systems. The results also show that the absolute value changes of the global natural frequency and the damping are very small in all four systems. By comparing the four systems in SP\&PS mode, when the size of the cylinder is the same, the maximum of the damping is bigger by the PZT than the PVDF ones, and the corresponding load resistance is smaller by the PZT than the PVDF ones. The maximum of the damping is the smallest in PP mode, meanwhile it is almost equal in SS mode and SP\&PS mode, when we compared System 1 in the different circuit combined modes.

The harvested power level drops quickly for some free stream velocities, and the maximum harvested power associated with the transverse displacement and the load resistance value. A comparison of the same size cylinder shows that the bigger harvested power can be obtained when the PZT is used. And a bigger harvested power is obtained in the bigger cylinder system when the same piezoelectric material is used. By comparing System 1 in different circuit combined modes, the maximum of the harvested voltage is the smallest one in PP mode and the biggest one in SS mode. The maximum of the harvested power is smallest in PP mode and almost the same in SS mode and SP\&PS mode, but the corresponding load resistance is much bigger in SS mode. All the results suggest the potential of using them for energy harvesting from VIV in water flow.

\section{Conflict of Interests}

The authors declare that there is no conflict of interests regarding the publication of this paper.

\section{References}

[1] P. Muralt, "Ferroelectric thin films for micro-sensors and actuators: a review," Journal of Micromechanics and Microengineering, vol. 10, no. 2, pp. 136-146, 2000.

[2] W. Zhou, W.-H. Liao, and W. J. Li, "Analysis and design of a selfpowered piezoelectric microaccelerometer," in Proceedings of SPIE Conference on Smart Structures and Materials 2005: Smart Electronics, MEMS, BioMEMS, and Nanotechnology (SPIE '05), vol. 5763, pp. 233-240, San Diego, Calif, USA, 2005.

[3] S. Roundy and P. K. Wright, "A piezoelectric vibration based generator for wireless electronics," Smart Materials and Structures, vol. 13, no. 5, pp. 1131-1142, 2004.

[4] D. J. Inman and B. L. Grisso, "Towards autonomous sensing," in Smart Structures and Materials 2006: Sensors and Smart Structures Technologies for Civil, Mechanical, and Aerospace Systems, Proceedings of SPIE, San Diego, Calif, USA, 2006.

[5] I. D. Capel, H. M. Dorrell, E. P. Spencer, and M. W. L. Davis, "The amelioration of the suffering associated with spinal cord injury with subperception transcranial electrical stimulation," Spinal Cord, vol. 41, no. 2, pp. 109-117, 2003.

[6] A. Erturk, W. G. R. Vieira, C. de Marqui Jr., and D. J. Inman, "On the energy harvesting potential of piezoaeroelastic systems," Applied Physics Letters, vol. 96, no. 18, Article ID 184103, 2010.

[7] C. De Marqui Jr., A. Erturk, and D. J. Inman, "Piezoaeroelastic modeling and analysis of a generator wing with continuous and segmented electrodes," Journal of Intelligent Material Systems and Structures, vol. 21, no. 10, pp. 983-993, 2010.

[8] C. de Marqui Jr., W. G. R. Vieira, A. Erturk, and D. J. Inman, "Modeling and analysis of piezoelectric energy harvesting from aeroelastic vibrations using the doublet-lattice method," Journal of Vibration and Acoustics, vol. 133, no. 1, Article ID 011003, 2011.

[9] A. Abdelkefi, A. H. Nayfeh, and M. R. Hajj, "Modeling and analysis of piezoaeroelastic energy harvesters," Nonlinear Dynamics, vol. 67, no. 2, pp. 925-939, 2012.

[10] A. Abdelkefi, A. H. Nayfeh, and M. R. Hajj, "Design of piezoaeroelastic energy harvesters," Nonlinear Dynamics, vol. 68, no. 4, pp. 519-530, 2012.

[11] A. Abdelkefi, A. H. Nayfeh, and M. R. Hajj, "Enhancement of power harvesting from piezoaeroelastic systems," Nonlinear Dynamics, vol. 68, no. 4, pp. 531-541, 2012.

[12] A. Abdelkefi, M. R. Hajj, and A. H. Nayfeh, "Sensitivity analysis of piezoaeroelastic energy harvesters," Journal of Intelligent Material Systems and Structures, vol. 23, no. 13, pp. 1523-1531, 2012.

[13] S. Pobering and N. Schwesinger, "A novel hydropower harvesting device," in Proceedings of the International Conference on MEMS, NANO and Smart Systems (ICMENS '04), pp. 480-485, August 2004.

[14] O. Doaré and S. Michelin, "Piezoelectric coupling in energyharvesting fluttering flexible plates: Linear stability analysis and conversion efficiency," Journal of Fluids and Structures, vol. 27, no. 8, pp. 1357-1375, 2011.

[15] S. Michelin and O. Doaré, "Energy harvesting efficiency of piezoelectric flags in axial flows," Journal of Fluid Mechanics, vol. 714, pp. 489-504, 2013.

[16] W. A. Mair and D. J. Maull, "Bluff bodies and vortex shedding-a report on Euromech 17," Journal of Fluid Mechanics, vol. 45, no. 2, pp. 209-224, 1971.

[17] O. M. Griffin, "Vortex-induced lift and drag on stationary and vibrating bluff bodies," Journal of Hydronautics, vol. 9, no. 4, pp. 160-164, 1975. 
[18] P. W. Bearman and J. M. R. Graham, "Vortex shedding from bluff bodies in oscillating flow: a report on Euromech 119," Journal of Fluid Mechanics, vol. 99, no. 2, pp. 225-245, 1980.

[19] P. W. Bearman, "Vortex shedding from oscillating bluff bodies," Annual Review of Fluid Mechanics, vol. 16, pp. 195-222, 1984.

[20] R. A. Skop and S. Balasubramanian, "A new twist on an old model for vortex-excited vibrations," Journal of Fluids and Structures, vol. 11, no. 4, pp. 395-412, 1997.

[21] D. Rockwell, "Vortex-body interaction," Annual Review of Fluid Mechanics, vol. 30, pp. 199-229, 1998.

[22] C. Evangelinos and G. E. Karniadakis, "Dynamics and flow structures in the turbulent wake of rigid and flexible cylinders subject to vortex-induced vibrations," Journal of Fluid Mechanics, vol. 400, pp. 91-124, 1999.

[23] A. Khalak and C. H. K. Williamson, "Investigation of relative effects of mass and damping in vortex-induced vibration of a circular cylinder," Journal of Wind Engineering and Industrial Aerodynamics, vol. 69-71, pp. 341-350, 1997.

[24] A. Khalak and C. H. K. Williamson, "Fluid forces and dynamics of a hydroelastic structure with very low mass and damping," Journal of Fluids and Structures, vol. 11, no. 8, pp. 973-982, 1997.

[25] A. Khalak and C. H. K. Williamson, "Motions, forces and mode transitions in vortex-induced vibrations at low mass-damping," Journal of Fluids and Structures, vol. 13, no. 7-8, pp. 813-851, 1999.

[26] R. Govardhan and C. H. Williamson, "Modes of vortex formation and frequency response of a freely vibrating cylinder," Journal of Fluid Mechanics, vol. 420, pp. 85-130, 2000.

[27] R. Govardhan and C. H. Williamson, "Resonance forever: existence of a critical mass and an infinite regime of resonance in vortex-induced vibration," Journal of Fluid Mechanics, vol. 473, pp. 147-166, 2002.

[28] R. Govardhan and C. H. K. Williamson, "Critical mass in vortex-induced vibration of a cylinder," European Journal of Mechanics-B/Fluids, vol. 23, no. 1, pp. 17-27, 2004.

[29] C. H. K. Williamson and R. Govardhan, "Vortex-induced vibrations," Annual Review of Fluid Mechanics, vol. 36, no. 1, pp. 413-455, 2004.

[30] R. N. Govardhan and C. H. K. Williamson, "Defining the "modified Griffin plot" in vortex-induced vibration: revealing the effect of Reynolds number using controlled damping," Journal of Fluid Mechanics, vol. 561, pp. 147-180, 2006.

[31] T. L. Morse and C. H. K. Williamson, "Employing controlled vibrations to predict fluid forces on a cylinder undergoing vortex-induced vibration," Journal of Fluids and Structures, vol. 22, no. 6-7, pp. 877-884, 2006.

[32] T. L. Morse and C. H. K. Williamson, "Prediction of vortexinduced vibration response by employing controlled motion," Journal of Fluid Mechanics, vol. 634, pp. 5-39, 2009.

[33] T. L. Morse and C. H. K. Williamson, "Steady, unsteady and transient vortex-induced vibration predicted using controlled motion data," Journal of Fluid Mechanics, vol. 649, pp. 429-451, 2010.

[34] A. Erturk and D. J. Inman, "An experimentally validated bimorph cantilever model for piezoelectric energy harvesting from base excitations," Smart Materials and Structures, vol. 18, no. 2, Article ID 025009, 2009.

[35] A. Abdelkefi, M. R. Hajj, and A. H. Nayfeh, "Phenomena and modeling of piezoelectric energy harvesting from freely oscillating cylinders," Nonlinear Dynamics, vol. 70, no. 2, pp. 1377-1388, 2012.
[36] A. Abdelkefi, M. R. Haji, and A. H. Nayfeh, "Power harvesting from transverse galloping of square cylinder," Nonlinear Dynamics, vol. 70, no. 2, pp. 1355-1363, 2012. 


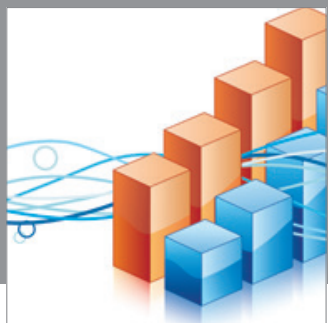

Advances in

Operations Research

mansans

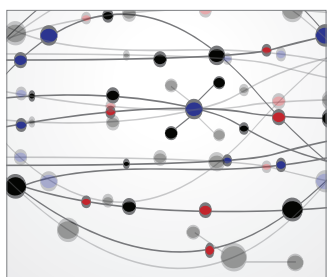

The Scientific World Journal
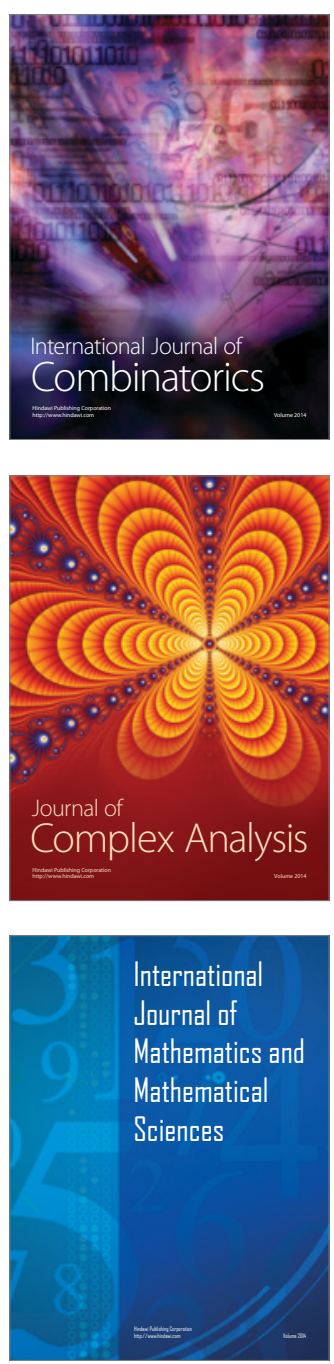
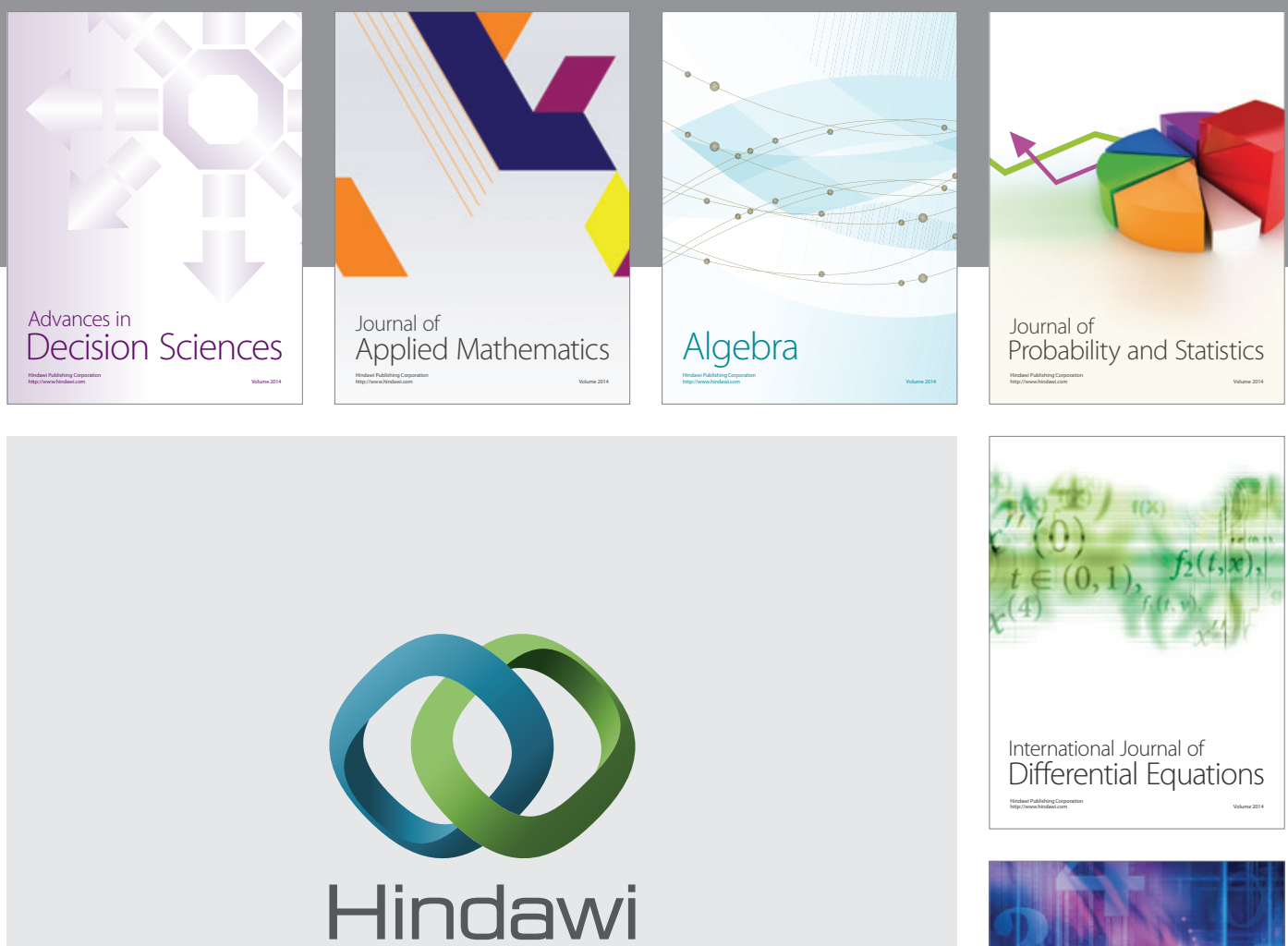

Submit your manuscripts at http://www.hindawi.com
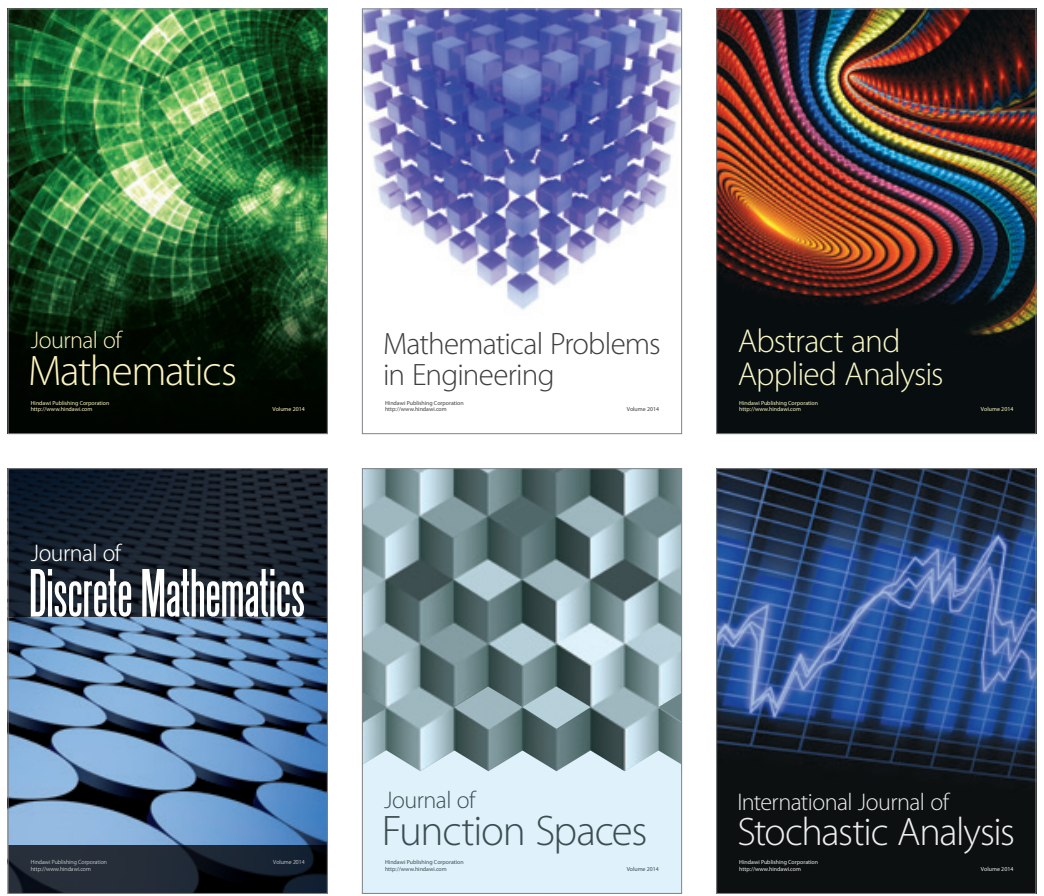

Journal of

Function Spaces

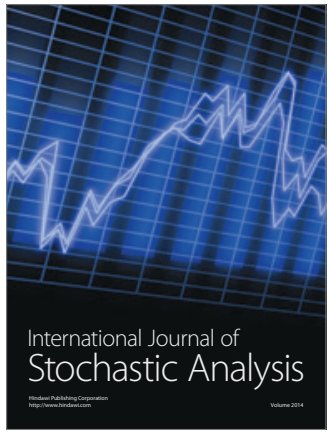

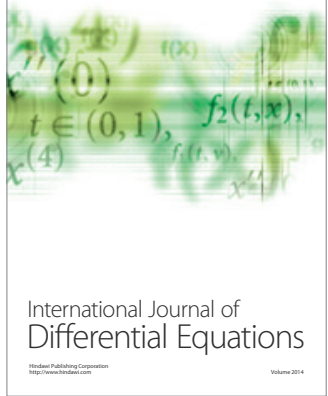
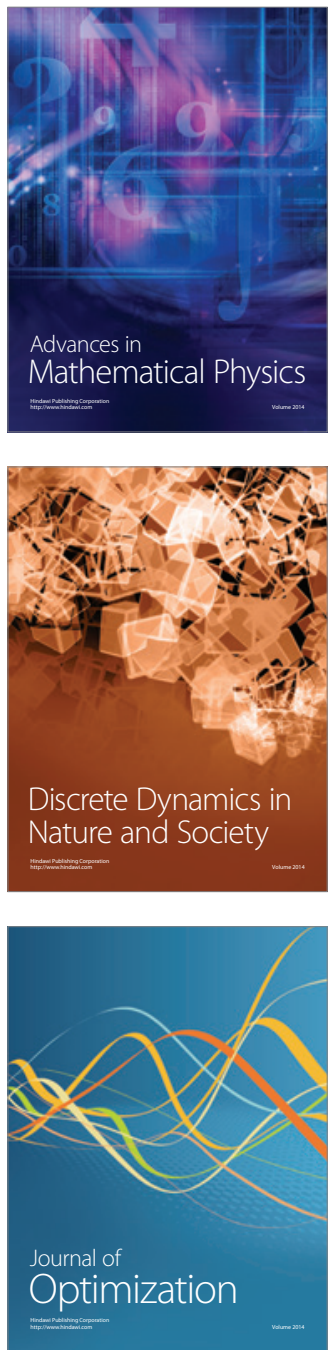